\title{
Primary Thyroid Lymphoma:A heterogeneous disease and presentation of two different cases
}

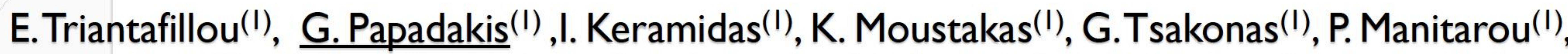

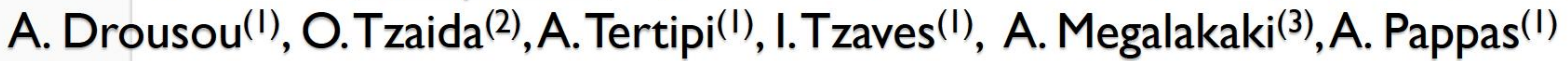 \\ Departments of 'Endocrinology, ${ }^{2}$ Pathology and ${ }^{3}$ Hematology, Anticancer Hospital of Piraeus "Metaxa"
}

Background: Primary thyroid lymphoma (PTL) is a rare disease representing $1-5 \%$ of all thyroid malignancies and $1 \%-2.5 \%$ of all lymphomas (I). Nevertheless, it frequently presents diagnostic and therapeutic challenges and given the heterogeneous nature of the disease, neither diagnosis nor treatment is always straightforward (2). We describe two patients with PTL with different clinical presentation, clinical picture and outcome.

Patients and Methods: Two female patients underwent total thyroidectomy. The first, a 67-year-old female patient, presented with a history of goiter since the age of 47 years old and on physical examination the thyroid was palpable and firm, with a prominent nodule of approximately $3 \mathrm{~cm}$ on the right lobe. FNAC of the large nodule, repeated twice in the past, showed features of Hashimoto thyroiditis (HT). The second patient, a 79-year-old female patient, presented with a large, painless thyroid mass accompanied by severe obstructive symptoms of the upper respiratory and gastrointestinal track. Thyroid function testing revealed subclinical hypothyroidism. The patient was afebrile and no weight loss or night sweats were reported. The laryngoscopy showed left vocal cord palsy. Biochemical parameters were within normal range. A thyroid ultrasound revealed a solid mass on the right lobe measuring $21 \times 26 \mathrm{~mm}$ and another solid mass on the left lobe measuring $26 \times 38 \mathrm{~mm}$ (Figure I). A fine needle aspiration cytology (FNAC) was performed and the cytologic analysis revealed follicular cells, immature lymphocytic elements and sparse colloid. Computed tomography (CT) of the neck confirmed the marked increase of the size of the thyroid gland, especially on the left lobe. The diameter of the trachea was decreased due to mass effect and neck nodes were enlarged.

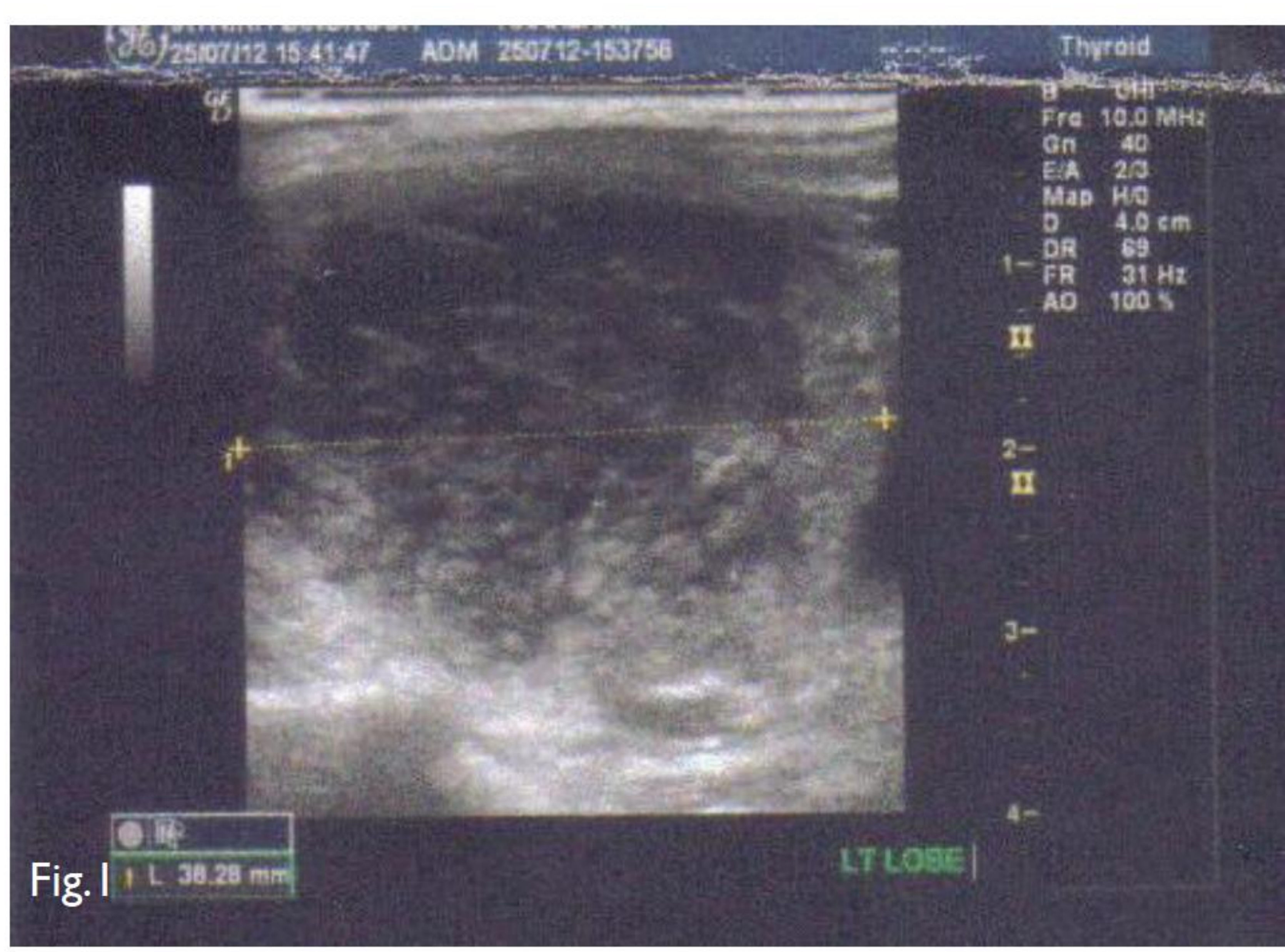

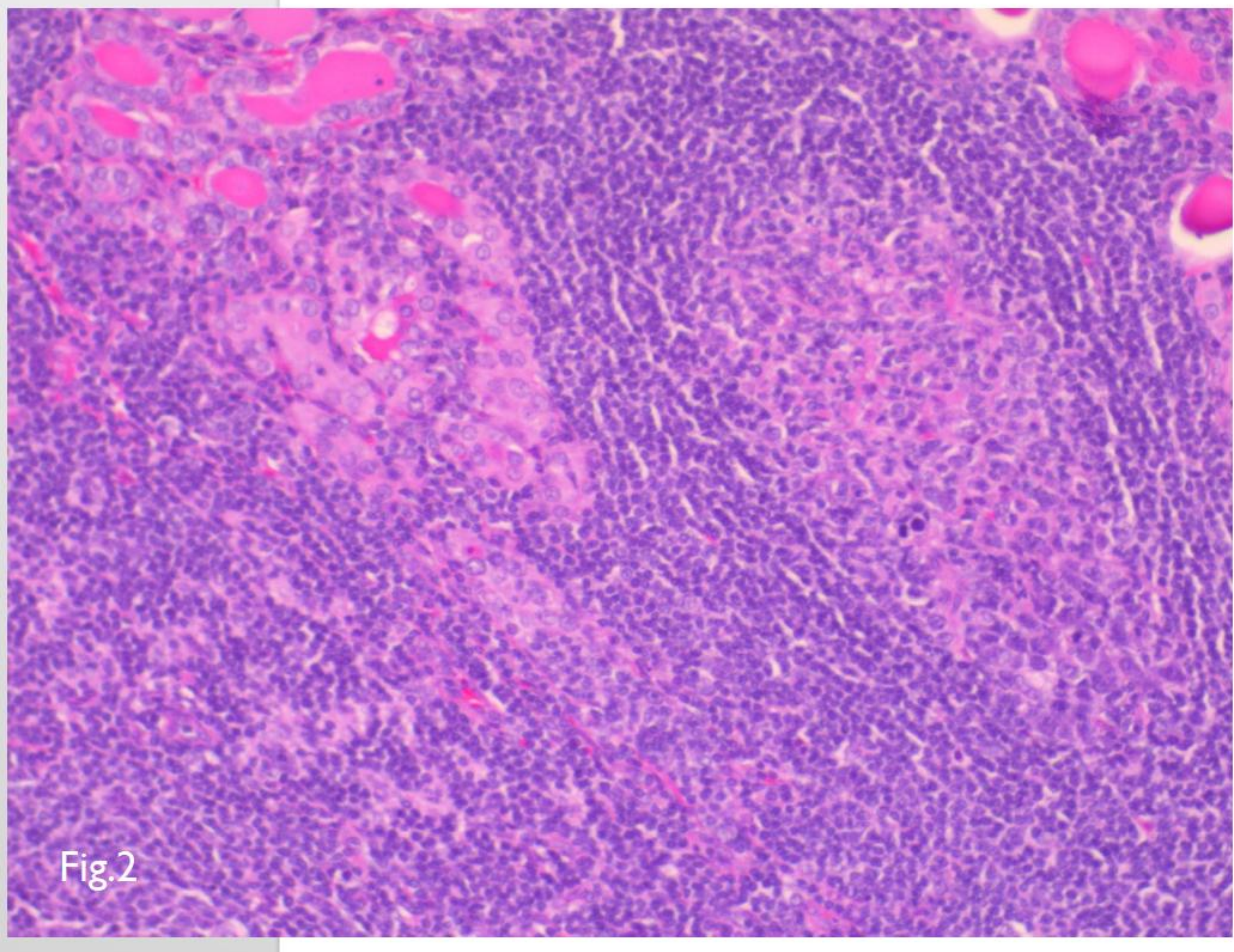

Results: Histology of the first patient revealed lymphocytic thyroiditis and a small focus of previously unsuspected PTL, coexistent with an occult papillary thyroid carcinoma. The palpable nodule corresponded to a hyperplastic nodule. Figure 2 shows the lymphoid infiltration of the thyroid follicle by neoplastic lymphoid cells and expansion of the marginal zone with H/E stain. The PTL was an incidental finding and two years after thyroidectomy she is free of disease without any further treatment.

Histology of the second patient confirmed the clinical suspicion of PTL and established the diagnosis of a diffuse large B-cell lymphoma. Figure 3 shows lymphoid infiltration of the thyroid follicle of this Bcell lymphoma in hematoxylin eosin staining (H/E). Staging was done according to the Ann Arbor staging system (3). CT of the thorax, abdomen and pelvis and bone marrow aspiration were negative for other neoplastic foci. The patient had stage IIE disease (involvement of the thyroid and lymph nodes on the same side of the diaphragm). Although she underwent standard chemotherapy, she died four months later.

Conclusions: Primary thyroid lymphoma may be suspected on the basis of clinical presentation. A rapidly growing painless goiter, frequently associated with obstructive phenomena, in a woman with HT in her sixth to seventh decade of life, is the typical presentation. Anaplastic thyroid carcinoma and sarcoma may also present as a painless rapidly growing thyroid mass and need to be included in the differential diagnosis. The most frequent histological subtype of PTL is the diffuse large B cell lymphoma accounting for approximately $70 \%$ of cases, followed by MALT lymphoma and follicular lymphoma that amount to $10 \%$ each. Other types, like small lymphocytic lymphoma, Burkitt's lymphoma, and Hodgkin's disease are less frequent (4). PTL may have an aggressive and lethal course on the one end of the spectrum and the other one may be quite indolent. Our two cases confirm the heterogeneous nature of PTL. References:

I) Widder S and Pasieka JL: Primary thyroid lymphomas. Curr Treat Options Oncol 5: 307-3|3, 2004

2) Thieblemont $C$ aser A, et al, Primary thyroid lymphoma is a heterogeneous disease. J Clin Endocrinol Metab 87: 105- III, 2002 2) Thieblemont C, Mayer A, et al, Primary thyroid lymphoma is a heterogeneous disease.

4) Graff-Baker A, Roman SA, Thomas DC, Udelsman R, and Sosa JA: Prognosis of primary thyroid lymphoma: demographic, clinical, and pathologic predictors of survival in I,408 cases. Surgery 146: I I05-I I I5, 2009

\begin{tabular}{|c|}
\hline 30 \\
\hline
\end{tabular}

